\title{
Case study: Saturday cognitive habilitation program for children with prenatal alcohol exposure
}

\author{
Molly N. Millians and Claire D. Coles \\ Emory University School of Medicine, Atlanta, Georgia, United States
}

\begin{abstract}
This case study describes the outcomes of a Saturday community intervention program for children suspected of or affected by prenatal exposure to alcohol who exhibited learning deficits. Five children participated in the program and received individualized interventions designed to address learning and academic deficits in either reading or mathematics. Often children affected by prenatal alcohol exposure exhibit deficits with executive processes, including metacognitive functioning, that interfere with learning. Instruction to improve metacognitive skills was incorporated into the intervention programs. The metacognitive training was adapted from the Math Interactive Learning Experience (MILE) and targeted the children's skills to plan, organize, shift, and evaluate problem solving strategies. Standardized tests of nonverbal reasoning and academic achievement were administered before and after the children received interventions to measure progress. The results indicated that four of the five children who participated in the program showed clinically significant gains with scores increasing from the borderline or low average to the average range on measures of nonverbal reasoning, reading comprehension, or mathematics reasoning. One child showed no gains on measures of nonverbal reasoning and reading. A variety of factors including age, cognitive profile, session attendance, and access to special education and other intervention services may have influenced the child's progress. Overall, the case reviews suggest that the interventions show promise to remediate learning problems of children affected by prenatal alcohol exposure in a community setting. Keywords: prenatal alcohol exposure, executive functioning, metacognitive functioning, cognitive habilitation.
\end{abstract}

Received 03 August 2013; received in revised form 23 December 2013; accepted 24 December 2013. Available online 28 April 2014.

\section{Introduction}

For more than 40 years, studies have shown that prenatal alcohol exposure is associated with physical abnormalities, or dysmorphia, growth delays, and neurocognitive impairments (Jacobson, Jacobson, Staton, Meintjes, \& Molteno, 2011; Davis, Desrocher, \& Moore, 2011). Due to neurodevelopmental delays, many children affected by prenatal alcohol exhibit deficits in adaptive functioning and learning, especially when interventions are not provided (Kodituwakku \& Kodituwakku, 2011). Research continues to examine the teratogenic effects of intrauterine alcohol exposure on development; yet few studies have investigated effective interventions to address learning deficits associated with prenatal alcohol exposure (Kodituwakku \& Kodituwakku, 2011; Bertrand, 2009; Kable, Coles, \& Taddeo, 2007).

Molly N. Millians and Claire D. Coles, Department of Psychiatry and Behavioral Sciences, Emory University School of Medicine. Correspondence regarding this article should be directed to: Molly N. Millians, Department of Psychiatry and Behavioral Science, Emory University, School of Medicine, 1256 Briarcliff Road, MSC/1256/001/1AV, Atlanta, Georgia, 30306. Phone: 4047129817 Fax: 4047129809. E-mail: molly.n.millians@emory.edu
The purpose of this case study is to describe outcomes from a Saturday, community-based intervention program for children suspected of or affected by prenatal alcohol exposure. Interventions incorporated training to improve higher level reasoning skills within remedial instruction to address deficits either reading or mathematics (Kable, Coles, \& Taddeo, 2007; Coles, Kable, \& Taddeo, 2009; Bertrand, 2009).

Neurodevelopmental studies have identified a range of functional deficits associated with prenatal alcohol exposure. These functional problems are found to have a neurological basis as supported by recent neuroimaging studies (Coles \& Li, 2011; Fryer et al., 2009; Wozniak, et al., 2009; Li et al., 2008). Cognitive impairments associated with prenatal alcohol exposure range from global intellectual delays to specific cognitive deficits. Many children affected by prenatal alcohol exposure exhibit global intellectual delays with scores below 70 on nationally standardized measures of intellectual abilities with scores between 85 and 115 as average or within the normal range (Sattler, 2004). Although not all children affected by prenatal alcohol exposure exhibit overall cognitive deficits, some exhibit specific impairments in areas of attention, encoding, working memory, visuospatial processing, decreased speed of processing, and or executive functioning that impact their academic performance, most notably in mathematics (Mattson, 
Crocker, \& Nguyen, 2011; Kable, Coles, \& Taddeo, 2007; Swanson, 2011).

Learning requires a closely linked network of separate cognitive processes that include attention, encoding, speed of information processing, working memory, and long-term memory (Butterworth \& Kovas, 2013; Bayliss, Jarrold, Baddeley, Gunn, \& Leigh, 2005; St. Clair-Thompson \& Gathercole, 2006). These cognitive processes select, transform, store, and retrieve information for further use. In addition to these processes, executive functioning contributes to efficient learning. For this case study, executive functioning is defined as a set of cognitive processes that contribute to the regulation of mental processes needed to complete complex tasks (St.Clair-Thompson \& Gathercole, 2006; Hofmann, Schmeichel, \& Baddeley, 2012). Specifically, executive functioning refers to the abilities to inhibit automatic or dominate responses, update incoming information, and to shift among tasks or mental processes (Hofmann, Schmeichel, \& Baddeley, 2012; Marcovitch \& Zelazo, 2009; Wu et al., 2012; Marcovitch \& Zelazo, 2009; St. Clair-Thompson \& Gathercole, 2006). Metacognition or the awareness of one's learning and the ability to allocate and regulate cognitive resources is included within processes associated with executive functioning (Hofmann, Schmeichel, \& Baddeley, 2012; Annervita \& Vauras, 2006). Often children affected by prenatal alcohol exposure exhibit deficits in one or more of these cognitive processes that interfere with learning and academic performance (St. ClairThompson \& Gathercole, 2006; Swanson, 2011; Kable, Coles, \& Taddeo, 2007; Mattson, Crocker, \& Nguyen, 2011; Ware et al., 2012; Kully-Martens, Treit, Pei, \& Rasmussen, 2013).

Studies have shown that children and adolescents affected by prenatal alcohol exposure score lower on tests of academic achievement, especially in mathematics, as compared to peers matched by age and cognitive abilities (Coles et al., 1991; Howell, Lynch, Platzman, Smith, \& Coles, 2006; Streissguth, et al., 1994). Also, children affected by prenatal alcohol exposure received lower teacher ratings in overall school performance, attending to class instruction, completing homework, and getting along with peers (Streissguth et al., 1994; Goldschmidt, Richardson, Cornelius, \& Day, 2004).

Although studies have shown that deficits associated with prenatal alcohol exposure impact learning and meeting the demands of school, minimal research has been completed to investigate interventions to address difficulties directly related to academic and school performance (Bertrand, 2009; Kable, Coles, \& Taddeo, 2007; Adnams et al., 2007). Two studies examined interventions for children affected by prenatal alcohol exposure that implemented metacognitive training within instruction to improve basic reading or mathematics (Adnams et al., 2007; Kable, Coles, \& Taddeo, 2007; Coles, Kable, \& Taddeo, 2009; Bertrand, 2009). A pilot study conducted in South Africa examined classroom interventions adapted from cognitive control therapy to improve metacognitive skills of 10 children of low socioeconomic status affected by prenatal alcohol exposure (Adnams et al., 2007; Riley et al., 2003). The interventions focused on increasing engagement to tasks, organizing thoughts, and awareness of actions. The participants showed no gains in overall academic skills. However, scores on post-test measures of behavior trended toward clinical significance. The children were reported to show improvements with regulating emotional reactions, cooperating with adults, and in their attitude and motivation toward school (Riley et al., 2003). The findings suggested that metacognitive training showed promise to improve classroom behaviors (Adnams et al., 2007; Riley et al., 2003; Kodituwakku \& Kodituwakku, 2011).

Researchers in the United States developed the Math Interactive Learning Experience (MILE) to address neurocognitive mathematical impairments and deficits in mathematics in children ages 3 through 10 affected by prenatal alcohol exposure. Interventions used in MILE were based on practices used in cognitive rehabilitation and early childhood education programs (Kable, Coles, \& Taddeo, 2007). Similar to models used by effective cognitive rehabilitation programs, MILE developed interventions to habilitate cognitive deficits within the context of a functional skill that are affected by the impairment (Ylvisaker et al., 2005; Kable, Coles $\&$ Taddeo, 2007). Interventions using this model are shown to increase generalization of skills (Kable, Coles \& Taddeo, 2007; Ylvisaker et al., 2005).

Specific strategies used in MILE were adapted from the High Scope/Perry Preschool program (Hohmann \& Weikart, 1995). That program was developed to study the long-term influence of a quality, educational program on the development of at risk, low income children. The program provided direct instruction to improve planning, decision making, concept development, and learning readiness skills. A longitudinal study comparing the outcomes of children who participated in the High Scope/Perry Preschool program showed improvements in educational and social responsibility as adults when compared to the control group (Weikart, 1998).

Due to deficits with efficient problem solving skills, children learned to use the "Plan-Do-Review Process" adapted from the High Scope/Perry Preschool Program (Kable, Coles, \& Taddeo, 2007; Mattson, Crocker, \& Nguyen, 2011; Hohmann \& Weikart, 1995). Children were taught to select relevant information, generate a plan to approach problems, monitor and adjust their problem solving techniques, and reflect upon the effectiveness of their strategies within the context of mathematics instruction (Kable, Coles, \& Taddeo, 2007; Bertrand, 2009).

Children assigned to the intervention group received 6 weeks of individualized instruction. Their caregivers were given information about neurocognitive deficits associated with the affects from prenatal alcohol exposure. Also, caregivers were provided weekly 
mathematic activities and directions on how to use the "Plan-Do-Review Process" when working with their children at home (Kable, Coles, \& Taddeo, 2007; Coles, Kable, \& Taddeo, 2009).

At follow-up, $58.6 \%$ of the children in the treatment group showed gains of one or more standard deviations on one of four tests of mathematic achievement; whereas only $23.1 \%$ of the controls showed such gains. Caregivers also reported decreases in their children's problematic behaviors (Bertrand, 2009; Kable, Coles, \& Taddeo, 2007; Coles, Kable, \& Taddeo, 2009). The results from the 6-month follow-up indicated that the participants maintained gains achieved in mathematics as well as improvements with behavior (Coles, Kable, \& Taddeo, 2009).

\section{Interventions from the Saturday cognitive habilitation program}

The Saturday cognitive habilitation program provided interventions for children suspected of or affected by prenatal alcohol exposure at risk for academic failure. The children in the Saturday program were between 10 and 13 years of age. The aims for the interventions were twofold. First, the program was designed to provide individualized interventions to address underlying cognitive deficits that impacted the children's academic performance in reading or in mathematics. Second, the program examined whether the interventions used in the MILE program could be adapted for use with older children to improve learning in a community-based setting (Hohmann \& Weikart, 1995; Kable, Coles, \& Taddeo, 2007). To improve awareness of learning and efficient problem solving, the "Plan-Do-Review Process" was adapted by teaching the children to use self-questioning techniques through each phase of the process (Perkins, Goodrich, Tishman, \& Owen, 1994). Examples of the guiding questions are presented in Table 1.

Table 1. Plan-Do-Review Questions

\begin{tabular}{ll}
\hline Phase & Questions \\
\hline \multirow{2}{*}{ Plan } & Do I know what to do? \\
& What looks familiar? \\
& Do I need to ask for more information? \\
& \\
Do & How am I doing? \\
& Do I need to change my strategy? \\
& \\
Review & How did I do? \\
& What do I need to remember for next time? \\
\hline
\end{tabular}

Due to deficits in cognitive shifting associated with prenatal alcohol exposure, the instructor used specific questioning methods to instigate transitions in thought processes (Mattson, Crocker, \& Nguyen 2011). The questioning techniques were similar to those used in studies examining children's readiness to change thought patterns (Siegler, 1995; Crowley, Shrager, \& Siegler, 1997; Siegler \& Chen, 2008). Siegler (1995) conducted a study examining cognitive change in 5-year-olds learning the concept of conservation. The results from the study indicated that the children who explained the examiner's perspective answered correctly more often than the children who were given only feedback by the examiner and children who were given feedback as well as explained how they solved a task. Siegler (1995) surmised that the children who were asked to provide an explanation from the examiner's perspective showed greater changes in learning.

Based upon Siegler's work, the children in the Saturday program were asked to explain how they solved a problem and state the reasons for the instructor's determining whether their solutions were correct or incorrect. If the children's answer was accurate, the instructor stated, "I agree with your answer. How do I know it is correct?" If answers were incorrect, the instructor would identify the error and ask, "Why do I think that is incorrect?" The questions were to create a shift in perspective in an attempt to increase cognitive flexibility. These instructional methods were created to address deficits with executive functioning that interfere with learning. The case report of the Saturday cognitive habilitation program is presented in the following sections.

\section{Case Report}

\section{Participants}

Five children affected by or suspected of prenatal exposure to alcohol and or other substances were enrolled in the Saturday cognitive habilitation program. All of the children resided within the metropolitan area of Atlanta, Georgia. The children resided with their current caregivers for more than 1 year. Records indicated that all of the children were involved with the Division of Family and Child Services (DFCS) in Georgia or in another state due to parental substance abuse or inability to care for their children's needs. Subsequently, two of the children had been adopted. Three of the children were in the care of relatives but remained under the guardianship of DFCS. All of the children received Medicaid, state and federally funded health insurance for children in foster care, individuals with disabilities, or families whose income is below the poverty level.

Referrals to the Saturday program were made by a clinic that provided evaluations and services for children affected by prenatal exposures to alcohol, drugs, and other toxins. All of the children were evaluated by the clinic's interdisciplinary team to examine whether concerns regarding the children's developmental delays and learning challenges would be associated with the effects from prenatal alcohol exposure or another developmental disorder or medical condition.

The interdisciplinary evaluation team consisted of a geneticist trained to discern the physical characteristics 
of prenatal alcohol exposure from other congenital birth defects or developmental disabilities, licensed clinical psychologists, a social worker, and a special educator to conduct testing and formulate a treatment plan. The interdisciplinary team followed the diagnostic criteria established by the Institute of Medicine (IOM) when evaluating the children for the effects from prenatal alcohol exposure (Stratton, Howe, \& Battaglia, 1996). A diagnosis of Fetal Alcohol Syndrome (FAS) was given when children had documented evidence of prenatal alcohol exposure, alcohol-related dysmorphia, height and/or weight below the national $10^{\text {th }}$ percentile, and evidence of neurodevelopmental delays not attributed to other medical factors. According to the IOM, it is permissible to give a diagnosis of FAS without documented evidence of prenatal alcohol exposure if a child presents growth retardation, alcohol-related dysmorphia, and cognitive impairments if no other genetic, medical, or developmental disorder would explain the physical anomalies and neurodevelopmental delays (Stratton, Howe, \& Battaglia, 1996). Children who had documented evidence of prenatal alcohol exposure, dysmorphia, and either growth deficits or neurodevelopmental delays were given a diagnosis of Partial Fetal Alcohol Syndrome (pFAS) (Stratton, Howe, \& Battaglia, 1996). No behavior problems were reported by the caregivers for four out of the five children. Child \#4 was reported by his caregiver to have behavior problems at home but not at school. A description of the children who participated in the Saturday cognitive habilitation program is presented in Table 2.

The program was funded by a gift to the clinic from the Spray Foundation, a private philanthropic organization. Services were provided at no cost to the families. The children who received services were neither part of a predetermined investigation nor an experimental study examining effective interventions; therefore, Institutional Review Board approval was not required. However, because the program was part of a clinic that provided medical services, procedures, to ensure consent for treatment and to protect sensitive health information as mandated by the Health Insurance Portability and Accountability Act of 1996 (HIPAA) 42 U.S.C.§1320d-9 were implemented.

\section{Program}

The cognitive habilitation program occurred for 20 Saturdays from September 2005 through May 2006. The program's calendar coincided with the local school system with breaks for Thanksgiving, December holidays, winter recess, and spring break. The instructor was a Georgia state certified special education teacher who received training regarding neurodevelopmental delays and secondary disabilities associated with prenatal exposure to alcohol and other substances. Sessions were provided on an individual basis. Each session was 60 minutes in length. The children received 50 minutes of direct instruction.
During the last 10 minutes of each session, caregivers were given feedback about their child's progress and recommendations for activities to be completed at home. The children varied in the number of sessions they attended due to illness, lack of transportation, and scheduling conflicts.

The type of intervention was determined by a review of each of the children's educational records and from concerns expressed by their caregivers during an interview. All of the children were administered a pretest to assess their academic function to guide treatment planning. Different measures of academic achievement were selected based upon caregiver concerns. Based upon the pre-test results, four of the children received interventions to address deficits in reading. One child received interventions for mathematics. All of the children received training using the "Plan-Do-Review Process" within their individualized programs. Posttests were administered during the last session of the program to determine if the children benefitted from the interventions.

\section{Measures}

The use of norm referenced tests allowed for a comparison of the children's scores to a sample of the population of the same age group (Sattler, 2004). The test scores were reported as composite scores with a mean of 100 and a standard deviation of 15 points. Scores between 85 and 115 are considered within normal limits with scores below 70 indicating deficient performance (Sattler, 2004).

All of the children were administered the Matrix Analogies Test, Expanded Form (MAT: EF) as part of the pre-test and post-test assessment (Naglieri, 1985). The MAT: EF was used to assess nonverbal reasoning, an area of deficiency often noted in children affected by prenatal alcohol exposure (Mattson, Crocker, \& Nguyen, 2011).

Two children were administered the Test of Reading Comprehension, Third Edition (TORC-3) to assess reading comprehension due to their age, grade level, and caregiver concerns about their reading skills (Brown, Hammill, \& Wiederholt, 1995). The TORC3 is a silent reading that assesses skills required in middle and upper grades. During the administration of the TORC-3, one child was noted to have problems with decoding and word recognition. The Test of Word Reading Efficiency (TOWRE) was administered to assess his fluent and accurate reading of sight words and non-words that follow common word patterns (Torgensen, Wagner, \& Rashotte, 1999). One child was administered the Gray Oral Reading Test, Fourth Edition to assess overall oral reading skills (Weiderholt $\&$ Bryant, 2001).

One child was referred to the program for difficulties with mathematics. The mathematics subtests from the Wechsler Individual Achievement Test, Second Edition (WIAT-II) were used to assess computation and applied mathematics (Psychological Corporation, 2001). All 
Table 2. Description of Participants

\begin{tabular}{|c|c|c|c|c|c|}
\hline Identification & $\# 1$ & $\# 2$ & $\# 3$ & $\# 4$ & $\# 5$ \\
\hline Age & $11 \mathrm{yrs} 1 \mathrm{mo}$ & 11 yrs 4 mo & $10 \mathrm{yrs} 6 \mathrm{mo}$ & 13 yrs 6 mo & 13 yrs $8 \mathrm{mo}$ \\
\hline Gender & Female & Female & Female & Male & Male \\
\hline Ethnicity & African-American & Caucasian & African-American & African-American & African-American \\
\hline $\begin{array}{l}\text { Prenatal alcohol } \\
\text { exposure }\end{array}$ & Suspected & Suspected & Confirmed & Confirmed & Confirmed \\
\hline $\begin{array}{l}\text { Alcohol-related } \\
\text { diagnosis }\end{array}$ & No & FAS & Deferred & FAS & pFAS \\
\hline $\mathrm{IQ}^{1}$ & 86 & 61 & 74 & 73 & 74 \\
\hline Verbal ability & 89 & 77 & 95 & 78 & 76 \\
\hline \multicolumn{6}{|l|}{ Nonverbal ability } \\
\hline & 88 & 60 & 57 & 70 & 61 \\
\hline Spatial $^{2}$ ability & 87 & 62 & 66 & N/A & 93 \\
\hline
\end{tabular}

of the children were administered either the reading or mathematics subtests from WIAT-II as part of the posttest battery.

Reliability coefficients for the tests ranged from .75 to .98 indicating moderate to high reliability across age groups. Also, the measures were reported to have adequate to high construct and content validity (McMorris, Rule, \& Steinberg, 1989; Green \& Perlman, 1998; Crumpton \& Miller-Whithead, 2003; Doll, Tindal, \& Nutter, 2003; Tindal \& Vacca, 2003).

\section{Definition of learning gains}

Clinically significant gains in learning are defined as an increase in performance from below normal on the pre-test to within the normal range or average on posttests (Jacobson \& Truax, 1991). Specifically, scores are considered to be clinically significant if there are increases of one or more standard deviations (15 points) with changes in the descriptive classification progressing toward average on the post-test as compared to the pretest (Kable, Coles, \& Taddeo, 2007).

\section{Cases}

A summary of the results for each child is presented below. Information regarding the children's scores from previous tests of cognitive abilities and related descriptive classifications are reported in Table 2 .
Child \#1.

Background. Child \#1 was an 11 year 1 month old African-American female. Prenatal alcohol exposure was suspected but not confirmed. According to a review of her records, she exhibited no evidence of alcohol-related dysmorphia or growth delays. Results from the psychological testing included in the interdisciplinary team evaluation indicated her cognitive functioning was within normal limits. Child \#1 had a history of academic difficulties, anxiety, and problems with attention. She was diagnosed with Unspecified Learning Disorder (LD-NOS) and Anxiety by the interdisciplinary team. Subsequently, she was diagnosed by her pediatrician with Attention DeficitHyperactivity Disorder (ADHD).

Education and intervention. Child \#1 attended sixth grade at a public school and received her instruction in the general education setting. She did not receive special education or early intervention services provided by her school system. Child \#1 was referred to the Saturday cognitive habilitation program to address concerns with mathematics and problem solving skills. She attended 13 of the 20 Saturday sessions. Based upon the results from the pre-test, interventions were developed to improve her understanding of the number system, recognizing the relationships between the mathematical operations of multiplication and division, and improving her skills to analyze and solve word problems. Instruction taught concepts of numbers in coordination with procedures to solve problems. Small 
blocks and other items were used to represent and work through mathematical problems prior to transforming the information into mathematical symbols. The "PlanDo-Review Process" was implemented throughout the instructional sessions.

Results. The Matrix Analogies Test, Expanded Form (MAT: EF) was administered to assess her nonverbal reasoning skills. The Numerical Operations and Math Reasoning subtests from the Wechsler Individual Achievement Test, Second Edition (WIAT-II) were administered to assess Child \#1's mathematical skills. The pre-test and post-test scores for Child \#1 are presented in Table 3.

Table 3. Pre-test and post-test Scores for Child \#1

\begin{tabular}{lll}
\hline Test & Pre-test & Post-test \\
\hline MAT: EF & 100 & 100 \\
WIAT-II & & \\
Math composite & 84 & 91 \\
Numerical operations & 92 & 91 \\
Math reasoning & 83 & 99 \\
\hline
\end{tabular}

According to the post-test findings, Child \#1 showed no gains in nonverbal reasoning. Scores from earlier testing and the pre-test indicated that Child \#1 exhibited an even pattern of cognitive development with verbal and nonverbal reasoning falling within normal limits. Her performance on the MAT: EF suggested that she did not exhibit deficiencies in nonverbal reasoning that required intervention.

Child \#1 made clinically significant gains in mathematical reasoning as shown by changes on her Math Reasoning subtest scores progressing from low average (83) on the pretest to average (99) on the posttest. However, she made no gains in basic computation. Similar to the findings from the MILE study, the results suggest that she benefitted from instruction to increase her awareness of the learning process and to attend to relevant information. She showed improvements in her skills to select and adjust strategies to solve a variety of mathematical problems. Additionally, the questioning techniques directed Child \#1 to represent mathematical information in different ways that contributed to improvements in applied mathematics (Crowley, Shrager, \& Siegler, 1997; Siegler, 1995; Kable, Coles, \& Taddeo, 2007).

Child \#2.

Background. Child \#2 was an 11 year 4 month old Caucasian female. Prenatal alcohol exposure was suspected but not confirmed. She exhibited alcohol-related dysmorphia, growth delays, and neurodevelopmental deficits. According to a review of previous testing, her cognitive abilities fell within the deficient to borderline range. Given the presence of alcohol-related dysmorphia, growth retardation, and cognitive impairments, she met criteria to receive a diagnosis of FAS without confirmed history of prenatal exposure (Stratton, Howe, \& Battaglia, 1996).
Education and intervention. Child \#2 was in the fifth grade and received special education services in a self-contained classroom at a public school. She received occupational therapy on a weekly basis at school. She had received special education services since entering elementary school. Child \#2 was referred to the Saturday cognitive habilitation program due to problems with abstract reasoning and reading comprehension. She attended 16 of the 20 Saturday sessions. The interventions focused on teaching Child \#2 to summarize information in her own words, recognize clues to identify relevant information, and synthesize information to develop conclusions or infer meaning. Self-questioning techniques were implemented within instruction to guide development of a framework to analyze the information.

Results. Child \#2 was administered the MAT: EF and the Test of Reading Comprehension, Third Edition (TORC-3) due to concerns with her reading comprehension for the pre-test. The MAT: EF and the reading subtests from the WIAT-II were administered for the post-test battery. The results from pre-tests and post-tests are provided in Table 4.

Table 4. Pre-test and post-test Scores for Child \#2

\begin{tabular}{lll}
\hline Test & Pre-test & Post-test \\
\hline MAT: EF & 79 & 82 \\
TORC-3 & & \\
Reading & 75 & \\
comprehension quotient & & \\
WIAT-II & & 94 \\
Reading composite & \\
Word reading & & 87 \\
Pseudoword reading & & 99 \\
Reading comprehension & 101 \\
\hline
\end{tabular}

Child \#2 showed no gains in her nonverbal reasoning. However, she made clinically significant gains in reading comprehension with test scores progressing from borderline (75) on the Reading Comprehension Quotient administered during the pretest to average (101) as shown by the Reading Comprehension subtest score from the post-test. The use of the "Plan-Do-Review Process" helped direct her attention to the relevant information. Child \#2 was observed to use the "Plan-Do-Review Process" independently as she analyzed information to respond to comprehension questions during the post-test.

Child \#3.

Background. Child \#3 was a 10 year 6 month old African-American female. Prenatal exposure to alcohol and crack cocaine was confirmed. Child \#3 exhibited normal growth at birth. She was significantly above average in height at the time of the interdisciplinary team evaluation. A review of her previous testing indicated Child \#3 exhibited an uneven pattern of cognitive development. Her verbal abilities fell within 
the average range. However, she exhibited deficient nonverbal and spatial functioning. Records indicated that she was suspected of having Hypomelanosis of Ito (HMI), a disorder associated with an $\mathrm{X}$ autosome translocation involving Xp11. In some cases, children with HMI exhibit abnormalities of the central nervous system that result in developmental delays (Retrieved on February 25, 2013, www.ncbi.nlm.nih.gov/omim). The diagnosis of FAS was deferred until additional medical testing could be completed (Stratton, Howe, $\&$ Battaglia, 1996). She was given a diagnosis of Unspecified Learning Disorder (LD-NOS). A diagnosis of Hypomelanosis of Ito was not confirmed at the time of her participation in the intervention program.

Education and intervention. Child \#3 was repeating third grade at a public school. She received instruction in a general education third grade classroom. According to a review of her records, Child \#3 also had repeated kindergarten. Her caregiver indicated that she exhibited learning problems since entering kindergarten. Child \#3 did not receive either early intervention or special education services. She was referred to the Saturday program due to concerns about her reading skills. Child \#3 attended 14 of the 20 Saturday sessions. The intervention plan for Child \#3 focused on improving word recognition, reading fluency, selecting relevant information from text, and summarizing the information. She received direct instruction to recognize common orthographic patterns to improve decoding and spelling. Interventions to improve her planning and problem solving skills were provided within the reading instruction.

Results. The MAT : EF and the reading subtests from the WIAT-II were administered for the pre-test and for the post-test. The results from the pre-test and post-test are presented in Table 5 .

Table 5. Pre-test and post-test Scores for Child \#3

\begin{tabular}{lll}
\hline Test & Pre-test & Post-test \\
\hline MAT: EF & 71 & 90 \\
WIAT-II & & \\
Reading composite & 72 & 83 \\
Word reading & 69 & 79 \\
Pseudoword reading & 75 & 75 \\
Reading comprehension & 80 & 102 \\
\hline
\end{tabular}

Child \#3 made clinically significant gains in nonverbal reasoning and in reading comprehension. Her scores on the MAT: EF increased from borderline (71) on the pre-test to average (90) on the post-test. Also, Child \#3 exhibited gains in reading comprehension with performance increasing from low average (80) on the Reading Comprehension subtest administered during the pre-test to average (102) on the subtest administered on the post-test. Additionally, scores suggest she exhibited some improvement with word recognition. She received a pre-test Word Reading subtest score that fell within the deficient range (69). Her post-test Word Reading subtest score fell within the borderline range (79).

Results indicated that she benefitted from the direct instruction to improve reasoning and reading comprehension. Her performance suggested instruction using the "Plan-Do-Review Process" improved her skills to select and link relevant information to support understanding (Crowley, Shrager, \& Siegler, 1997). Previous cognitive testing indicated average verbal abilities but deficient nonverbal and spatial functioning. It is surmised that Child \#3 was able to use language to mediate and compensate for deficits in nonverbal and spatial reasoning to support learning (Crowley, Shrager, \& Siegler, 1997; Siegler \& Chen, 2008). It is important to note that she had not received special education or intervention services prior to attending the Saturday cognitive habilitation program; thus, the increase of scores may be associated with the initial receipt of interventions.

\section{Child \#4.}

Background. Child \#4 was a 13 year 6 month old African-American male. A review of his records indicated prenatal alcohol exposure was confirmed. He exhibited alcohol-related dysmorphia and growth delays at birth. Previous psychological testing indicated that his cognitive abilities fell within the borderline range of functioning. Child \#4 received the diagnosis of FAS (Stratton, Howe, \& Battaglia, 1996). During an interview, the caregiver reported that Child \#4 exhibited behavior problems at home. His caregiver indicated that he ignored household rules, refused to complete daily chores, and disregarded attempts to correct his behavior.

Education and intervention. Child \#4 was in the eighth grade at a public school. He received special education instruction for reading, language arts, and mathematics in classes co-taught by a special education teacher and a general education teacher. Records indicated that he received special education services since kindergarten. He was referred to the Saturday program due to concerns about his reading comprehension. He attended 12 of the 20 Saturday sessions. His intervention program was designed to improve sight word knowledge, word attack skills, and comprehension of text presented on his independent reading level. His instructional program implemented a phonetic-based approach to teach sound-to-symbol associations to address weaknesses with decoding and spelling skills. Controlled vocabulary texts that corresponded with the level of decoding instruction were used when working with reading comprehension. Training using the "Plan-Do-Review Process" to increase his attention and awareness to the learning process was provided throughout the sessions.

Results. The MAT: EF was administered as part of the pre-test and post-test batteries. Because of concerns with his reading skills and his age, the TORC-3 was administered. However, during the pre-test he was noted to have significant difficulties decoding the passages. 
The Test of Word Reading Efficiency (TOWRE) was administered to assess word recognition and phonemic decoding fluency. The reading subtests from the WIATII were administered as part of the post-test. Table 6 provides the pre-and-post-test scores for Child \#4.

\begin{tabular}{|c|c|c|}
\hline Test & Pre-test & Post-test \\
\hline MAT: EF & 80 & 94 \\
\hline \multicolumn{3}{|l|}{ TORC-3 } \\
\hline $\begin{array}{l}\text { Reading } \\
\text { Comprehension Quotient }\end{array}$ & 52 & \\
\hline \multicolumn{3}{|l|}{ TOWRE } \\
\hline Total Word Reading Efficiency & 56 & \\
\hline Sight Word & 66 & \\
\hline Phonemic Decoding & 61 & \\
\hline \multicolumn{3}{|l|}{ WIAT-II } \\
\hline Reading Composite & & 51 \\
\hline Word Reading & & 50 \\
\hline Pseudoword Reading & & 68 \\
\hline Reading Comprehension & & 59 \\
\hline
\end{tabular}

The results indicate that Child \#4 showed improvements in nonverbal reasoning that trended toward a clinically significant gain. Child \#4 received a pre-test score on the MAT: EF that fell within the low average range (80) and a post-test score falling within the average range (94). The increase in scores suggests Child \#4 benefitted from the training using the "PlanDo-Review Process" and questioning techniques. He demonstrated increased attention to the stimuli and integration of the information to support nonverbal reasoning (Crowley, Shrager, \& Siegler, 1997).

However, Child \#4 showed no gains in his word reading or word attack skills. He attended a total of 12 sessions. At times he did not attend consecutive weekly sessions. His reported behavior problems at home may have influenced his attendance and his motivation during the sessions. Given his age, cognitive profile, and severity of his reading difficulties, he would benefit from an increase in the frequency of sessions over a longer duration to address his learning deficits.

\section{Child \#5.}

Background. Child \#5 was a 13 year 8 month old African-American male. According to a review of his records, prenatal exposure to alcohol was confirmed. He exhibited alcohol-related dysmorphia but no growth delays. Previous testing indicated his overall intellectual functioning, verbal, and nonverbal reasoning skills fell within the deficient to borderline range. However, his spatial abilities were described as falling in the average range. Child \#5 received a diagnosis of pFAS due to meeting three of the four criteria for a diagnosis of FAS (Stratton, Howe, \& Battaglia, 1996).

Education and intervention. Child \#5 was in the eighth grade and attended a charter school under the auspices of the local school system. He received instruction in a special education class of approximately six children for reading, language arts, mathematics, science, and social studies. He received both speech and language therapy and occupational therapy on a weekly basis at school. In addition, Child \#5 participated in weekly tutoring sessions at school. He received special education services since third grade. Child \#5 was referred to the Saturday program due to concerns about his reading skills. He attended 17 of 20 Saturday sessions. His intervention program utilized a phonetic based approach to improve decoding skills. Also, direct instruction was used to increase his skills to recognize common words, word attack strategies, reading fluency, and to infer information from text. His reading instruction followed the methods used in his special education program at school to provide consistency. He received direct instruction on using the "Plan-DoReview Process" during each session.

Results. The MAT: EF was administered as part of the pre-test and post-test batteries. Due to concerns about his reading skills, the Gray Oral Reading Test, Fourth Edition (GORT-4) was administered during the pre-test. The reading subtests from the WIAT-II were administered to measure changes after receiving interventions. The results from Child \#5's pre-test and post-tests are presented in Table 7.

Table 7. Pre-test and post-test Scores for Child \#5

\begin{tabular}{lll}
\hline Test & Pre-test & Post-test \\
\hline MAT: EF & 90 & 95 \\
GORT-4 & & \\
Oral Reading & 58 & \\
Quotient & & \\
WIAT-II & \\
Reading Composite & 69 \\
Word Reading & 61 \\
Pseudoword Reading & 79 \\
Reading Comprehension & 75 \\
\hline
\end{tabular}

Child \#5's scores on the MAT: EF pre-test (90) and post-test (95) fell within the average range; therefore, he did not exhibit deficits in nonverbal reasoning that required intervention. The results indicated that Child \#5 made no gains in reading. Multiple factors such as his age, cognitive profile, and the severity of his learning difficulties may have influenced his progress. It also is possible that the interventions did not address his specific reading difficulties. The use of the "Plan-DoReview Process" may not be beneficial when attempting to remediate rote skills such as word recognition or reading fluency (Crowley, Shrager, \& Siegler, 1997).

Records indicated that Child \#5 received interventions prior to and during his participation in the Saturday program. It is possible that he exhibited an increase of skills when he first received interventions with a slowing of improvements as he continues to require support to address residual deficits (Fay et al., 2010; Anderson, Catroppa, Morse, Haritou, \& Rosenfeld, 2009). No behavior problems were reported 
by his caregiver, his teachers, or noted during the intervention sessions.

Child \#5's caregiver reported improvements in his verbal expression of ideas. This was not evaluated using standardized measures for the program. Follow-up was not possible given the short duration of the Saturday program.

\section{Discussion}

A review of the cases suggests that training to improve elements of executive functioning within instruction to address academic deficits shows potential to remediate learning difficulties of children affected by prenatal alcohol exposure. The outcomes indicate that four out of the five children who participated in the Saturday cognitive habilitation program showed clinically significant gains in learning and academics. Gains were made in as few as 12 sessions. Similar to findings from studies examining efficacy of MILE, the children in the Saturday program benefitted from the interventions to increase problem solving skills and awareness of the learning process (Kable, Coles, \& Taddeo, 2007; Adnams et al., 2007; Siegler, 1995). The results suggest that the strategies used in MILE could be adapted for use with older children to address learning problems in a community-based program.

The children made clinically significant gains in reading comprehension, mathematics reasoning, and nonverbal reasoning but not in word reading or recall of mathematical facts. For example, Child \#4 and Child \#5 received interventions to address deficits with decoding, reading fluency, and sight word recognition. Neither child showed changes in word reading scores after receiving interventions. Although other factors such as the appropriateness of the interventions, age, and the severity of cognitive and learning deficits may have impacted their progress, the metacognitive training did not influence improvements in their basic reading skills. Additionally, Child \#1 showed gains in applied mathematics, an area requiring abstract reasoning, but not in arithmetic. Studies have suggested that metacognition contributes to flexible representation of information that can be integrated and adjusted to solve problems (Crowley, Shrager, \& Siegler, 1997; Siegler \& Chen, 2008). Other interventions are needed to improve automatic recall of information (Siegler \& Chen, 2008). Based upon the outcomes, careful consideration of cognitive processes in relation to the context of a skill is required when developing interventions.

A review of the cases suggested that children's individual cognitive profiles influenced benefits received from the interventions. For example, earlier cognitive testing indicated that Child \#3 exhibited verbal abilities that fell within the average range as compared to age-matched peers. The results from the post-test indicated that she showed greater overall gains with improvements in both nonverbal reasoning and reading comprehension as compared to the other children. Her adequate language abilities supported the generalization of strategies (Spitz, Ponsford, Rudzki, Mailer, 2012; Fay et al., 2010). Further investigation into the impact of individuals' cognitive profiles that may predict responses to interventions is warranted (Jonsson, Catroppa, Godfrey, Smedler, \& Anderson, 2013; Catroppa et al., 2009; Kodituwakku \& Kodituwakku, 2011).

Research has suggested that individuals with traumatic brain injury (TBI) who have higher cognitive abilities, less severe and complex brain injury, and adequate executive functioning showed greater recovery after receiving interventions (Spitz, Ponsford, Rudzki, \& Maller, 2012; Fulton, Yeates, Taylor, Walz, \& Wade, 2012; Catroppa et al., 2009). The cases reflected a similar pattern of recovery. Children who exhibited global intellectual impairments showed fewer gains than the children who had cognitive functioning within normal limits or adequate language abilities. It is likely that children affected by prenatal alcohol exposure who exhibit similar cognitive impairments as children with acquired brain damage would show a similar recovery pattern (Mattson, Crocker, \& Nguyen, 2011; Spitz, Ponsford, Rudzki, \& Maller, 2012). Cross-disability studies are needed to investigate similarities among disorders and responses to interventions to develop effective methods to address deficits associated with prenatal alcohol exposure.

There are limitations to the case study. The descriptions from the cases may not be used to draw conclusions about effective interventions for the larger population of children affected by prenatal alcohol exposure. Experimental studies with a control group and time point testing using consistent standardized measures would be needed. Controlled studies would need to conduct follow-up testing after 6 months, 1 year, and/or as children progress through grade levels to determine if the interventions had lasting effects. Also, minimal information was gathered about interventions received at school or from private providers that may have influenced the outcomes. Studies would need to gather information about the range of services children received while participating in an intervention program. This is to investigate possible interactions among programs. In addition, information regarding the amount of time caregivers worked with their children was not considered. Studies would need to consider the impact of caregiver involvement when developing interventions. Regardless of the limitations, the findings suggest that that individualized programs designed to remediate underlying cognitive deficiencies within context of a skill has potential to become a model for effective interventions for children affected by prenatal alcohol exposure.

Children affected by prenatal alcohol can exhibit a range of cognitive deficits including impairments with executive functioning. The children in these case studies received direct instruction to improve cognitive flexibility, problem solving, and metacognitive skills, all aspects of executive functioning. Following intervention, the children made gains in areas of nonverbal reasoning, 
applied mathematics, and reading comprehension that require abstract reasoning and efficient problem solving skills rather than automatic recall of basic information. These outcomes are encouraging and suggest that learning and academic deficits associated with the effects from prenatal alcohol exposure can be addressed by appropriate interventions.

\section{Acknowledgement}

We would like to thank the Spray Foundation and the children and their families who participated in the program. Also, we would like to thank Elles Taddeo, Mary Ellen Lynch, Karen Howell, Julie Kable, and David Millians for their assistance preparing this paper.

\section{References}

Adnams, C.M., Sorour, P., Kalberg, W.O., Kodituwakku, P., Perold. M.D., Kotze, A., September, S., Castle, B., Gossage, J., May, P.A. (2007). Language and literacy outcomes from a pilot intervention study for children with fetal alcohol spectrum disorders in South Africa. Alcohol, 41, 403-414. doi:10.1016/j.alcohol.2007.07.005

Anderson, V., Catroppa, C., Morse, S., Haritou, F., \& Rosenfeld, J.V. (2009). Intellectual outcome from preschool traumatic brain injury: A 5-year prospective, longitudinal study. Pediatrics, 124, e1064-e1071. doi: 10.1542/peds.2009-0365

Annevirta, T., \& Vauras, M. (2006). Developmental changes of metacognitive skill in elementary school children. The Journal of Experimental Education, 74(3), 195-226. doi:10.3200/ JEXE.74.3.195226

Bayliss. D.M., Jarrold, C.J., Baddeley, A.D., Gunn, G.M., \& Leigh, E. (2005). Mapping the developmental constraints on working memory span performance. Developmental Psychology, 41(4),579597.doi: $10.1037 / 0012-1649.41 .4 .579$

Bertrand, J. (2009). Interventions for children with fetal alcohol spectrum disorders (FASDs): Overview of the findings for five innovative research projects. Research in Developmental Disabilities, 30, 986-1006. doi:10.1016/j.ridd.2009.02.003

Brown, V.L., Hammill, D.D., \& Wiederholt, J.L. (1995). Test of Reading Comprehension, Third Edition. Austin, TX: Pro-ed.

Burden, M.J., Westerlund, A., Mucjle, G., Dodge, N., Dewailly, E., Nelson, C.A., ... \& Jacobson, J.L. (2011). The effects of maternal binge drinking during pregnancy on neural correlates of response inhibition and memory in childhood. Alcoholism: Clinical and Experimental Research, 35(1), 69- 82.doi:10.1111/j.15300277.2010.01323.x

Butterworth, B., \& Kovas, Y. (2013). Understanding neurocognitive developmental disorders can improve education for all. Science, 340, 300-305. doi: 10.1126/science. 1231022

Catroppa, C., Anderson, V.A., Muscara, F., Morse, S.A., Haritou, F., Rosenfeld, J., \& Heinrich, L.A. (2009). Education skills: Longterm outcome and predictors following paediatric traumatic brain injury. Neuropsychological Rehabilitation, 19(5), 716-732. doi:10.1080/09602010902732868

Coles, C.D., Brown, R.T., Smith, I.E., Platzman, K.A., Erickson, S., \& Falek, A. (1991). Effects of prenatal alcohol exposure at school age. I. Physical and cognitive development. Neurotoxicology and Teratology, 13, 357-367.

Coles, C.D., Kable, J.A., \& Taddeo, E. (2009). Math performance and behavior problems in children affected by prenatal alcohol exposure: Intervention and follow-up. Journal of Developmental \& Behavioral Pediatrics, 30(1), 7-15.doi:10.1097/ DBP.0b013e3181966780

Coles, C.D., \& Li, Z. (2011). Functional neuroimaging in the examination of effects of prenatal alcohol exposure. Neuropsychological Review, 21, 119-132. doi:10.1007/s11065011-9165-y

Crowley, K., Shrager, J., \& Siegler, R.S. (1997). Strategy discovery as a competitive negotiation between metacognitive and associative mechanisms. Developmental Review, 17, 462-489. Retrieved from http://www.psy.cmu.edu/ siegler/crowetal97.pdf

Crumpton, N.L., \& Miller Whithead, M. (2003). Review of the Gray Oral Reading Test, Fourth Edition. In B.S. Plake, J.C. Impara, \& R.A. Spies (Eds.). The fifteenth mental measurements yearbook [electronic version]. Retrieved from http://buros.unl. edu/buros

Davis, K., Desrocher, M., \& Moore, T. (2011). Fetal alcohol spectrum disorders: A review of neurodevelopmental findings and interventions. Journal of Developmental and Physical Disability, 23, 143-167. doi:10.1007/s10882-010-9204-2

Doll, B.J., Tindal, G., \& Nutter, M. (2003). Review of the Wechsler Individual Achievement Test, Second Edition. In B.S. Plake, J.C. Impara, \& R.A. Spies (Eds.). The fifteenth mental measurements yearbook [electronic version]. Retrieved from http://buros.unl.edu/ buros/

Elliot, C.D., Hale, J.B., Fiorello, C.A., Dorvil, C., \& Moldovan, J. (2010). Differential ability scales-ii prediction of reading performance: Global scores are not enough. Psychology in the Schools, 47(7), 698-720. doi:10.1002/pits.20499

Ewing-Cobbs, L., Barnes, M., Fletcher, J.M., Levin, H.S., Swank, P.R., \& Song, J. (2004). Modeling of longitudinal academic achievement scores after pediatric traumatic brain injury. Developmental Neuropsychology, 25(1-2), 107-133. doi:10.1080/87565641.2004. 9651924

Fay, T.B., Yeates, K.O., Taylor, H.G., Bangert, B., Dietrich, A., Nuss, K.E., Rusin, J., \& Wright, M. (2010).Cognitive reserve as a moderator of postconcussive symptoms in children with complicated and uncomplicated mild traumatic brain injury. Journal of the International Neuropsychological Society, 16(1), 94-105. doi:10.1017/S1355617709991007

Fulton, J.B., Yeates, K.O., Taylor, H.G., Walz, N.C., \& Wade, S.L., (2012). Cognitive predictors of academic achievement in young children 1 year after traumatic brain injury. Neuropsychology, 26 (3),314-322. doi:10.1037/a0027973

Fryer, S.L., Schweinsburg, B.C., Bjorkquist, O.A., Frank, L.R., Mattson., S.N., Spadoni,A.D., \& Riley, E.P.(2009). Characterization of white matter microstructure in fetal alcohol spectrum disorders. Alcoholism: Clinical and Experimental Research, 33(3), 1-8. doi:10.1111/j.1530-0277.2008.00864.x

Goldschmidt, L., Richardson, G.A., Cornelius, M.D., \& Day, N.L. (2004). Prenatal marijuana and alcohol exposure and academic achievement at age 10. Neurotoxicology and Teratology, 26, 521532. doi:10.1016/jntt.2004.04.003

Green, F.J., \& Perlman, C. (1998). Test review of the Test of Reading Comprehension, Third Edition. In J.C. Impara, \& B.S. Plake, (Eds.). The thirteenth mental measurements yearbook [electronic version]. Retrieved from http://buros.unl.edu/buros/

Hofmann, W., Schmeichel, B.J., \& Baddeley, A.D. (2012). Executive functions and self- regulation. Trends in Cognitive Sciences, 16(3), 174-180. doi: 10.1016/j.tics.2012.01.006

Hohmann, M., \& Weikart, D.P. (1995). The high/scope plan-doreview process. Educating young children (pp.167-243). Ypsilanti, MI: High/Scope Press.

Howell, K. K., Lynch, M.E., Platzman, K.A., Smith, H.G., \& Coles, C.D. (2006). Prenatal alcohol exposure and ability, academic achievement, and school functioning in adolescence: A longitudinal follow-up. Journal of Pediatric Psychology, 31(1), 116-126. doi:10.1093/jpepsy/jsj029

Jacobson, N.S., \& Truax, P. (1991). Clinical significance: A statistic approach to defining meaningful change in psychotherapy research. Journal of Consulting and Clinical Psychology, 59(1), 12-19.

Jacobson, S.W., Jacobson, J.L., Stanton, M.E., Meintjes, E.M., \& Molteno, C.D. (2011). Biobehavioral markers of adverse effect in fetal alcohol spectrum disorders. Neuropsychological Review, 21, 148-166. doi:10.1007/s11065-011-9169-7

Jonsson, C.A., Catroppa, C., Godfrey, C., Smedler, A.-C., \& Anderson, V. (2013). Cognitive recovery and development after traumatic brain injury in childhood: A person-oriented longitudinal study. Journal of Neurotrauma, 30, 76-83. doi:10.1089/neu.2012.2592

Kable, J.A., Coles, C.D., \& Taddeo, E. (2007). Socio-cognitive habilitation using the math interactive learning experience program for alcohol-affected children. Alcoholism: Clinical and 
Experimental Research, 31(8), 1425-1434. doi:10.1111/j.15300277.2007.00431.x

Kodituwakku, P.K., \& Kodituwakku, E.L. (2011). From research to practice: An integrative framework for the development of interventions for children with fetal alcohol spectrum disorders. Neuropsychological Review, 24, 204-223. doi: 10.1007/s11065011-9170-1

Kully-Martens, K., Treit, S., Pei, J., \& Rasmussen, C., (2013). Affective decision-making on the iowa gambling task in children and adolescents with fetal alcohol spectrum disorders. Journal of the International Neuropsychological Society, 19, 137-144. doi:10.1017/S1355617712001026

Li, Z., Coles, C.D., Lynch, M.E., Ma, X., Peltier, S., \& Hu, X. (2008). Occipital-temporal reduction and sustained visual attention deficit in prenatal alcohol exposed adults. Brain Imaging and Behavior, 29, 39-48. doi:10.1007/s11682-007-8013-0

Marcovitch, S. \& Zelazo, P.D. (2009). A hierarchical competing systems model of the emergence and early development of executive function. Developmental Science, 12(1), 1-25. doi:10.1111/j.14677687.2008.00754.x

Mattson, S.N., Crocker, N., \& Nguyen, T.T. (2011). Fetal alcohol spectrum disorders: Neuropsychological and behavioral features. Neuropsychological Review, 21, 81-101. doi:10.1007/s11065-0119167-9

McMorris, R.F., Rule, D.L., \& Steinberg, W.J. (1989). Review of the Matrix Analogies Test. In J.C. Conoley \& J.J. Kramer (Eds.). The tenth mental measurements yearbook [electronic version]. Retrieved from http://buros.unl.edu/buros/

Naglieri, J. (1985). The Matrix Analogies Test, Expanded Form. San Antonio, TX: Psychological Corporation.

Online Mendelian Inheritance in Man, OMIM ${ }^{\circledR}$ Johns Hopkins University (2011). Hypomelanosis of Ito. (MIM: 30037). Retrieved from http://omim.org/

Perkins, D.N., Goodrich, H., Tishman, S., \& Owen, J.M. (1994). Thinking connections, learning to think \& thinking to learn. Menlo Park, CA: Addison-Wesley.

Riley, E.P., Mattson, S.N., Li, T-K., Jacobson, S.W., Coles, C.D., Kodituwakku, P.K., ... \& Korkman, M.I. (2003). Neurobehavioral consequences of prenatal alcohol exposure: An international perspective. Alcoholism: Clinical and Experimental Research, 27(2), 362-373. doi:10.1097/01.ALC.0000052703.38558.B2

Sattler, J.M. (2004). Useful statistical and measurement concepts. Assessment of children, cognitive applications (4th $\mathrm{ed} ., \mathrm{pp} .86-127)$. La Mesa, CA: Jerome M. Sattler, Publisher, Inc.

Siegler, R.S. (1995). How does change occur: A microgenetic study of number conservation. Cognitive Psychology, 28, 225-273. Retrieved from http://www.psy.cmu.edu/ siegler/siegler95.pdf

Siegler, R.S., \& Chen, Z. (2008). Differentiation and integration: Guiding principles for analyzing cognitive change. Developmental Science, 11(4), 433-453. doi: 10. 1111/j.1467-7687.2008.00689.x

Spitz, G., Ponsford, J.L., Rudzki, D., \& Maller, J.J. (2012). Association between cognitive performance and functional outcome following traumatic brain injury: A longitudinal multi-level examination. Neuropsychology, 26(5), 604-612. doi: 10.1037/a0029239

St.Clair-Thompson, H.L. \& Gathercole, S.E. (2006). Executive functions and achievements in school: Shifting, updating, inhibition, and working memory. The Quarterly Journal of Experimental Psychology, 59(4), 745-759. doi:10.1080/17470210500162854
Stratton, K., Howe, C., \& Battaglia, F.C. (Eds.) (1996). Fetal alcohol syndrome: Diagnosis, epidemiology, prevention, and treatment. Washington, D.C.: National Academy Press.

Streissguth, A.P., Barr, H.M., Carmichael Olsen, H., Sampson, P.D., Bookstein, F.L., \& Burgess, D.M. (1994). Drinking during pregnancy decreases word attack and arithmetic scores on standardized tests: Adolescent data from a population-based prospective study. Alcoholism: Clinical and Experimental Research, 18(2), 248-254.

Swanson, H.L. (2011). Working memory, attention, and mathematical problem solving: A longitudinal study of elementary school children. Journal of Educational Psychology, 103(4), 821-837. doi:10.1037/a0025114

The Psychological Corporation (2001). Wechsler Individual Achievement Test, Second Edition. San Antonio, TX: Psychological Corporation.

Tindal, G., \& Vacca, J.J. (2003). Review of the Test of Word Reading Efficiency. In B.S. Plake, J.C. Impara, \& R.A. Spies (Eds.). The fifteenth mental measurements yearbook [electronic version]. Retrieved from http://buros.unl.edu/buros/

Torgensen, J.K., Wagner, R.K., Rashotte, C.A. (1999). Test of Word Reading Efficiency. Austin, TX: Pro-ed.

Vaurio, L., Riley, E.P., \& Mattson, S.N., (2008). Differences in executive functioning in children with heavy prenatal alcohol exposure or attention-deficit/hyperactivity disorder. Journal of the International Neuropsychological Society, 14, 119-129. doi: $10.1017 / \mathrm{S} 1355617708080144$

Ware, A.L., Crocker, N., O’Brien, J.W., Deweese, B.N., Roesch, S.C., Coles, C.D., ... Mattson, S.N., \& CIFASD (2012). Executive function predicts adaptive behavior in children with histories of prenatal alcohol exposure and attention-deficit/hyperactivity disorder. Alcoholism: Clinical and Experimental Research, 36(8), 1431-1441. doi:10.1111/j.1530-0277.2011.01718.x

Weikart, D. (1998). Changing early childhood development through educational intervention. Preventive Medicine, 27,233-237. Retrieved from http://www.journals.elsevier.com/preventive-medicine/

Wiederholt, J.L., \& Bryant, B.R. (2001). Gray Oral Reading Test, Fourth Edition. Austin, TX: Pro-ed.

Wozniak, J.R., Muetzel, R.L., Mueller, B.A., McGee, C.L., Freerks, M.A., Ward, E.E., ... \& Lim, K.O. (2009). Microstructural corpus callosum anomalies in children with prenatal alcohol exposure: An extension of previous diffusion tensor imaging findings. Alcoholism: Clinical and Experimental Research, 33(10), 18251835. doi:10.1111/j.1530-0277.2009.01021.x

Wozniak, J.R., \& Muetzel, R. L. (2011). What does diffusion tensor imaging reveal about the brain and cognition in fetal alcohol spectrum disorders. Neuropsychological Review, 21, 133-147. doi:10.1007/s11065-011-9162-1

Wu, K.K., Chan, S.K., Leung, P.W., Liu, W-S, Leung, F.L.T., Ng, R. (2011). Components and developmental differences of executive functioning for school-aged children. Developmental Neuropsychology, 36(3), 319-337. doi:10.1080/87565641.2010.549979

Ylvisaker, M., Adelson, P.D., Braga Willandino, L., Burnett, S.M., Glang, A., Feeney, T., Moore, W., Rumney, P., \& Todis, B. (2005). Rehabilitation and ongoing support after pediatric TBI. Twenty years of progress. Journal of Head Trauma Rehabilitation, 20(1), 95-109. Retrieved from http://journals.lww.com/headtraumarehab/ pages/issuelist.aspx?year $=2005$ 